\title{
FEM type method for reconstruction of plane stress tensors from limited data on principal directions
}

\author{
J. Irša \& A. N. Galybin \\ Wessex Institute of Technology, Southampton, UK
}

\begin{abstract}
This paper presents a method for reconstruction of maximum shear stress and stress trajectories from discrete data on principal directions. The domain is divided into smaller subdomains where stress potentials are assumed to be linear holomorphic functions. The functions obey continuity along element interfaces, which is used to form the first group of equations. The known data on principal directions are used in the second group of equations. Therefore, no stress magnitudes are involved in formulations, which eventually leads to a homogeneous system of linear algebraic equations. In order to make the system inhomogeneous an extra equation is added. It represents mean value of maximum shear stress over the domain. The reconstructed maximum shear stress, therefore, includes an arbitrary positive multiplicative parameter.
\end{abstract}

Keywords: photoelasticity, stress trajectories, holomorphic function.

\section{Introduction}

Numerical method presented in this paper aims to reconstruct maximum shear stresses from discrete data on principal directions. Several examples from photoelasticity $[1,2]$ are considered to verify effectiveness of the method.

In the past photoelasticity was widely used in mechanics of solids [1,2]. It is an optical method to determine stresses in plane models on the basis of birefringence, the property, observed in certain transparent material. The optical properties of such materials are modified with intensity of loading. The normal incidence of light is resolved into two components, each one coinciding with a principal plane of stress. Placing photoelastic sample between polarizers whose axes are perpendicular, one can observe black fringes in the sample, these are referred to as isoclinics. Orientations of the two principal stresses are obtained by 
rotating the polarizers from $0^{\circ}$ to $90^{\circ}$, and drawing the isoclinics. The stress trajectory presents a curve the tangents to which coincide with the principal direction of one of the principal stresses, therefore the pattern of stress trajectories is further drawn by using the obtained set of discrete stress orientations.

The procedures for obtaining stress trajectories and determination of stresses from the photoelastic data, so called process of stress separation, are detailed in textbooks $[1,2]$. They are based on relatively dense set of uniformly distributed data, which allows one to determine the stress field with high accuracy and even to consider this field as a continuous one.

In geophysics, the data on stress orientations in the earth's crust can be obtained by different experimental methods (see the WSM project for detail [3]). However these data are significantly inhomogeneous and irregular. Moreover, the data are mostly related to the tectonic plate margins and large regions of the crust are not populated with the data at all. This structure of the data makes it impossible to directly transfer the methods used in photoelasticity to the problem of stress identification in the lithosphere. Therefore special approaches have recently been developed in [4-6]. These approaches make use of mathematical theory of plane elasticity [7], where the mean stress and deviatoric stress functions are described by means of two holomorphic functions, complex potentials. A method for reconstructing maximum shear stress field from discrete data on stress trajectories, using approximation of holomorphic functions across whole domain has firstly been applied in [4]. In this paper an example from photoelasticity for a beam under three point bending test is presented to verify the effectiveness of the proposed approach. It is further applied for geophysical data to study stresses in the Australian continent $[4,5]$. In these papers single plane domains have been considered. The method has also been applied for a pair of adjacent elastic regions to study elastic stress field of Antarctica plate, where the most data are known on the continent boundaries [6]. Apart from data on stress orientations, the continuity of the stress vector across the margins of the plate has been used in this paper as an additional conditions imposed on the sought solutions.

The present paper presents a method where the domain is divided into smaller subdomains and approximation is performed within each subdomain with implied continuity of approximating functions across the subdomain boundaries. Similar method has recently been developed for harmonic problem of heat flux reconstruction from temperature data [8].

The method is further verified with synthetic data, as well as with experimental data extracted from photoelasticity experiments.

\section{The method}

\subsection{Equation of plane elasticity}

In $2 \mathrm{D}$ stress field, the plane symmetric stress tensor is given by its components $\sigma_{11}, \sigma_{12}, \sigma_{22}$. The stress tensor can be described by means of the following stress functions [7]: 


$$
P(z, \bar{z})=\frac{1}{2}\left(\sigma_{11}+\sigma_{22}\right), \quad D(z, \bar{z})=\frac{1}{2}\left(\sigma_{22}-\sigma_{11}\right)+i \sigma_{12}
$$

Functions $P$ and $D$ represent the mean stress and the stress deviatoric respectively. The mean stress function is a real-valued function and the deviatoric stress is a complex valued function of complex conjugated variables $z=x+i y, \bar{z}=x-i y$. The functions $P$ and $D$ satisfy equations of equilibrium that can be expressed in the following complex form:

$$
\frac{\partial P}{\partial z}=\frac{\partial D}{\partial \bar{z}}
$$

The deviatoric stress function $D$ can be written in the form:

$$
D(z, \bar{z})=\tau_{\max }(z, \bar{z}) e^{i \alpha(z, \bar{z})}
$$

Here the modulus of function $D$ presents the maximum in-plane shear stress $\tau_{\max }=|D|$ and $\alpha=\arg (D)$ can be expressed as follows:

$$
\alpha=\pi-2 \varphi
$$

where $\varphi$ is the principal direction.

For homogenous isotropic elastic medium following equation is satisfied:

$$
\Delta P=0
$$

The stress functions $P$ and $D$ according to the Kolosov-Muskhelishvili formulae [7] can be expressed in terms the form:

$$
P(z, \bar{z})=\Phi(z)+\overline{\Phi(z)}, \quad D(z, \bar{z})=\bar{z} \Phi^{\prime}(z)+\Psi(z)
$$

where the complex potentials $\Phi(z)$ and $\Psi(z)$ are holomorphic functions.

\subsection{Problem description}

Let $\Omega$ be a simply connected elastic domain. It is assumed that principal directions $\varphi_{j}$ are known as a discrete set of points $z_{j}(\mathrm{j}=1 \ldots N)$, located within the domain. The task is to reconstruct the stress field from principal directions, in particular, the stress deviator, presented by the function $D$.

The data on principal directions have no restrictions on the type of distribution. In examples considered in section 3 synthetic data are uniformly distributed while photoelastic data are not uniform.

It has been shown in [4] that the reconstruction of stress trajectories is unique, while the solution of $\tau_{\max }$ contains one multiplicative positive constant, and therefore the solution for $P$ contains an extra additive real parameter caused by integration of (3). We further concentrate on the reconstruction of the stress deviator only and therefore do not pay much attention to the additive free parameter.

The domain is divided into $n$ smaller subdomains of an arbitrary shape. In every subdomain, the holomorphic functions entering in the second equation (6) are approximated by linear holomorphic functions are specified as follows:

$$
\begin{aligned}
& \Phi^{(m)}(z)=a_{0}^{(m)}+a_{1}^{(m)} z \\
& \Psi^{(m)}(z)=a_{2}^{(m)}+a_{3}^{(m)} z
\end{aligned}, \quad m=1 \ldots n
$$


$a, b, c$ and $d$ are unknown complex constants, therefore 8 unknown real constants can be associated with every single subdomain. Principal directions are associated with the stress deviators in subdomains, i.e. the arguments of the functions $D^{(m)}(z, \bar{z})=\bar{z} \Phi^{\prime(m)}(z)+\Psi^{(m)}(z)$, therefore the arguments of complex potentials cannot be specified separately.

\subsubsection{Discretization}

The discretization of the domain and introduction of collocation points on its interfaces are performed with respect to the examples considered in section 3 . Rectangular elements are used for the rectangular domain and polar elements are used for circular ring domain (Figure 1).
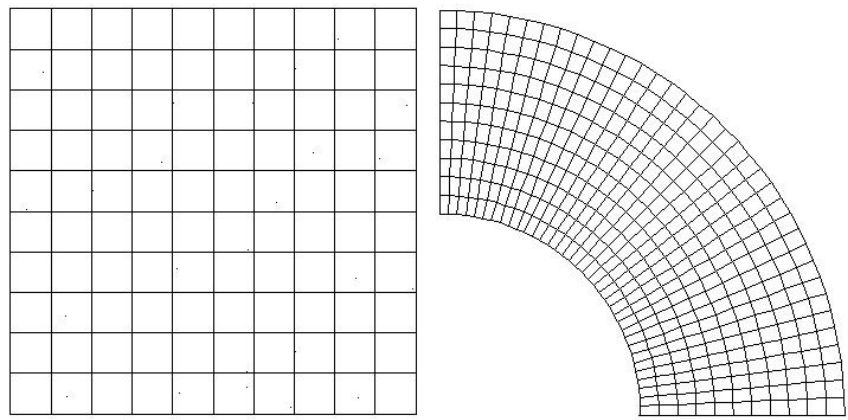

Figure 1: Rectangular element type (left) and polar element type (right).

\subsubsection{Equations of continuity, condition equations and non-homogenous equation}

In is assumed that stresses are continuous in $\Omega$. Thus, the approximating holomorphic functions, representing $\Phi^{\prime}(z)$ and $\Psi(z)$ obey continuity across interfaces at collocation points. For the $k$-th collocation point, lying on the interface between the elements numbered $m$ and $m+1$, the equations of continuity for $\Phi^{\prime}(z)$ and $\Psi(z)$ are as follows:

$$
\begin{aligned}
& \Phi^{\prime(m)}\left(z_{k}\right)-\Phi^{\prime(m+l)}\left(z_{k}\right)=0, m=1 \ldots n ; \quad k=1 \ldots N_{C P} \\
& \Psi^{(m)}\left(z_{k}\right)-\Psi^{(m+l)}\left(z_{k}\right)=0
\end{aligned}
$$

As mentioned above these two equations do not include information about the principal directions and therefore the following expression for the stress deviators is used to impose data:

$$
D^{(m)}(z, \bar{z})=\left(a_{0} \bar{z}+a_{1} z\right) \bar{z}+a_{2}+a_{3} z
$$

The maximum shear stress is a real valued function; therefore the following equations are valid in every subdomain:

$$
\operatorname{Im}\left[\left(a_{0}^{(m)} \bar{z}_{j}+a_{1}^{(m)} z_{j} \bar{z}_{j}+a_{2}^{(m)}+a_{3}^{(m)} z_{j}\right) e^{-i \alpha_{j}}\right]=0, j=1 \ldots N
$$

This equation is satisfied for every known location $z_{j}$. 
A non-homogenous equation has to be added to the system to address the fact that the field of $\tau_{\max }$ has one multiplicative arbitrary parameter. It is further assumed that the average value of $\tau_{\max }$ over the whole region as unity, which leads to the following equation:

$$
\sum_{m=1}^{N_{\text {elem }}}\left[\left(a_{0}^{(m)} \bar{z}_{j}+a_{1}^{(m)} z_{j} \bar{z}_{j}+a_{2}^{(m)}+a_{3}^{(m)} z_{j}\right) e^{-i \alpha_{j}}\right]=N_{\text {elem }}
$$

The total system consists of equations given by (8), (10) and (11).

By extracting the real and imaginary parts of equations (8), (10) and (11) the real system of linear algebraic equations (SLAE) can be obtained and rewritten in a matrix form:

$$
\mathbf{A x}=\mathbf{b}
$$

where $\mathbf{A} \in R^{m x n}, \mathbf{b} \in R^{m}$ and $m>n . \boldsymbol{x}$ is the vector of the unknown real coefficients, of the length $n$. The vector $\boldsymbol{x}$ is composed of real and imaginary parts of unknown complex coefficients. Vector $\boldsymbol{b}$ is known exactly, while, $\boldsymbol{A}$ the matrix of the SLAE depends on data quality, type and size of element. The matrix $\mathrm{A}$ is not a square matrix, the system is over-determined and therefore the left-hand side $\boldsymbol{A x}$ does not exactly equal to $\boldsymbol{b}$ and thus the system is inconsistent. However an approximate solution, $\mathbf{x}^{*}$, can be found by the least squares method [9] that minimises the residual to bring the error below the given level:

$$
\|\mathbf{A x} *-\mathbf{b}\|_{2} \leq \varepsilon
$$

where $\|. . .\|_{2}$ stands for the $L_{2}$ norm.

If the system is well-posed and not large, then the inversion of the matrix does not meet difficulties and the approximate solution takes the form:

$$
\mathbf{x}^{*}=\left(\mathbf{A}^{T} \mathbf{A}\right)^{-1} \mathbf{A}^{T} \mathbf{b}
$$

The condition number $(C N)$ is used in the numerical examples to control wellposedness of the SLAE.

\section{Results}

The method was first tested for a simple synthetic example in order to verify the accuracy. Other tests include data compiled from photoelastic experiments for rectangular and polar domains.

\subsection{Results for synthetic example}

Let potentials $\Phi^{\prime}$ and $\Psi$ be known in $\Omega$. Given these, one can calculate the stress deviator and obtain maximum shear stress and stress trajectories. Synthetic data extracted from the given potentials are referred to as the ideal data.

The potentials are chosen as low degree polynomials:

$$
\begin{aligned}
& \Phi^{\prime}(z)=0.2+(0.2+i 0.4) z \\
& \Psi(z)=-0.1+i 0.5+(-0.4+i 0.1) z+(-0.4+i 0.7) z^{2}
\end{aligned}
$$


Computational domain is the square $-1<x<1$ and $-1<y<1$. One singular point is found inside of this area, which refers zero maximum shear stress.

Within this area 36 uniformly distributed data on principal directions and 64 rectangular elements with 2 collocation points in every interface have been used (Figure 2). The system consisting of 933 equations and 512 unknowns produced $\mathrm{CN}=422$ and residual equal to 5.7 .

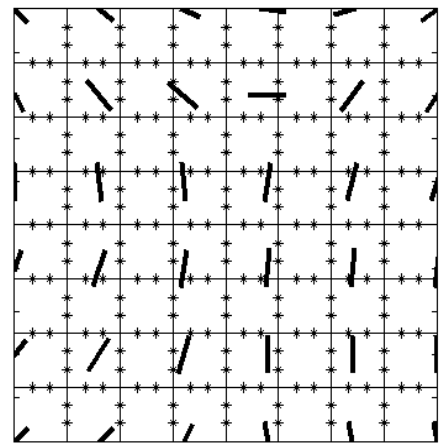

Figure 2: Computational domain showing subdomains, data on principal directions and collocation points.

The results of reconstruction are compared with the characteristics of the ideal stress field given by (15); Figure 3 presents contour map of the reconstructed and ideal maximum shear stress stresses; Figure 4 shows the profiles of maximum shear stresses over several cross sections. Ideal and reconstructed stress trajectory fields are plotted in Figure 5, which illustrates to illustrate that the singular point has been identified with high accuracy.
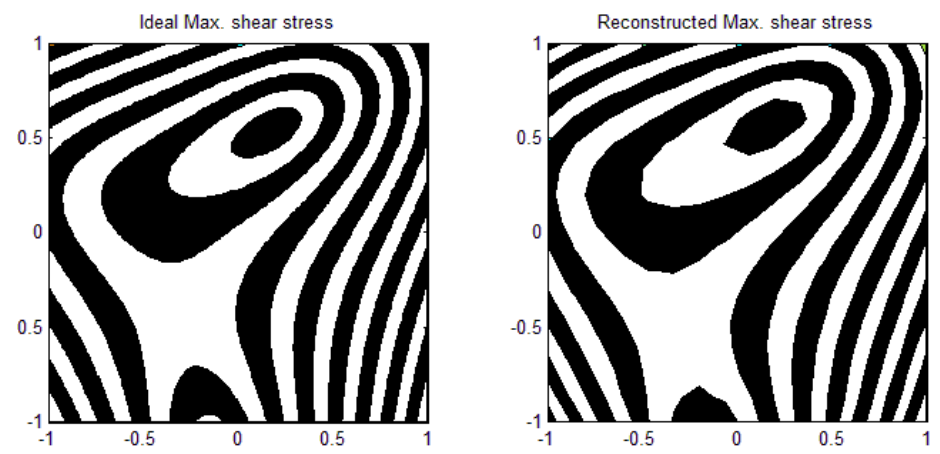

Figure 3: $\quad$ Ideal (left) and reconstructed (right) maximum shear stress

\subsection{Results for gas turbine blade under thermal shock}

A photoelastic experiment on stress determination in a gas turbine blade caused by of thermal shock [2] is used to collect 300 data subjected to errors, Figure 6. 
Mesh Reduction Methods 63
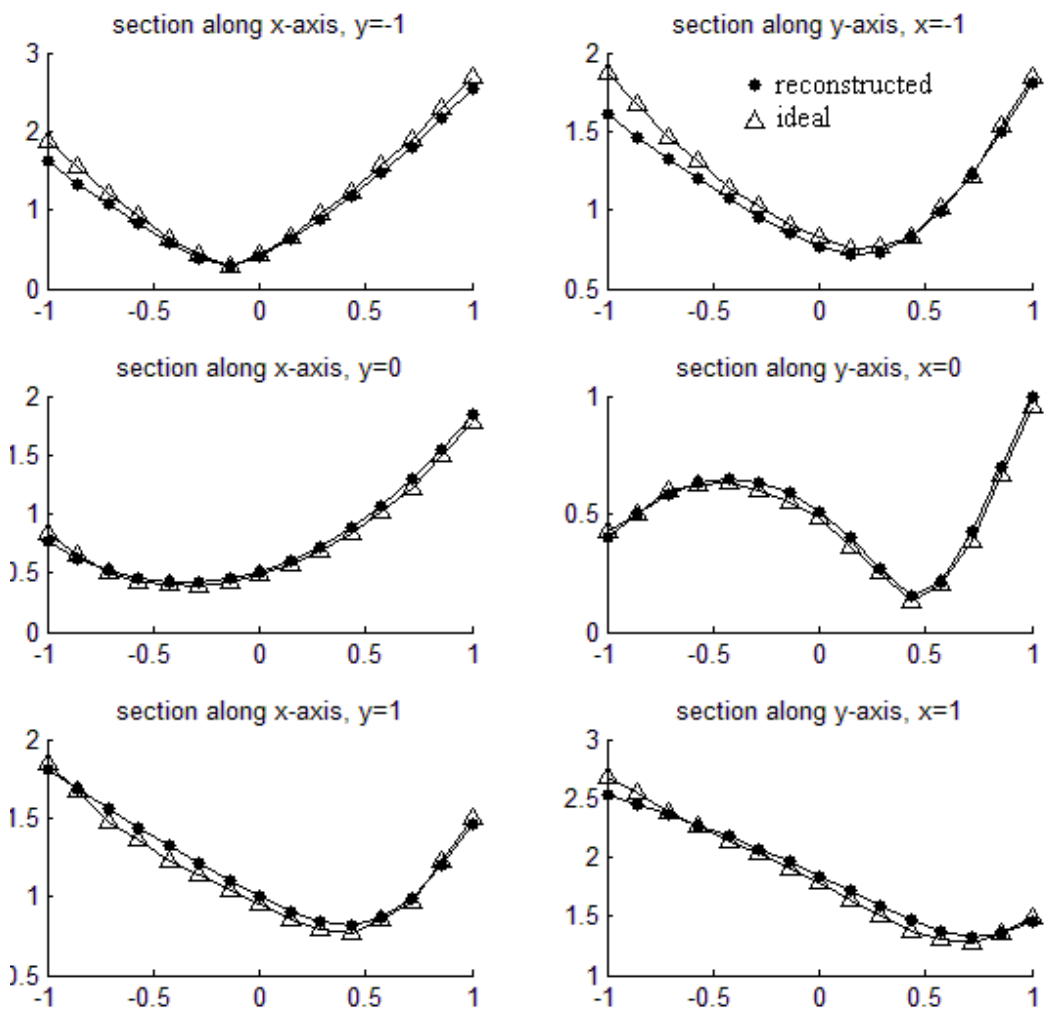

Figure 4: $\quad$ Profiles of reconstructed (dots) and ideal (triangles) maximum shear stress.
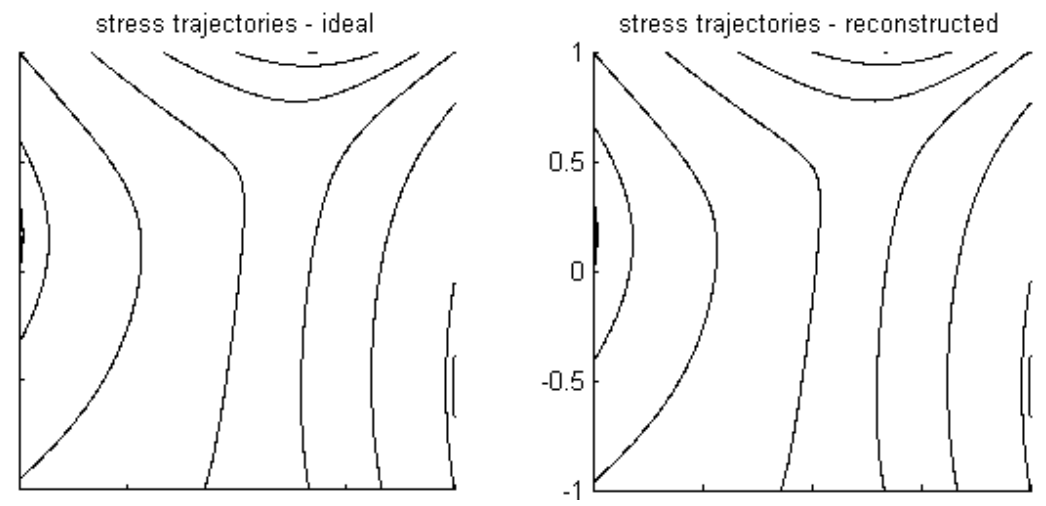

Figure 5: $\quad$ Stress trajectories, ideal (left) and reconstructed (right). 


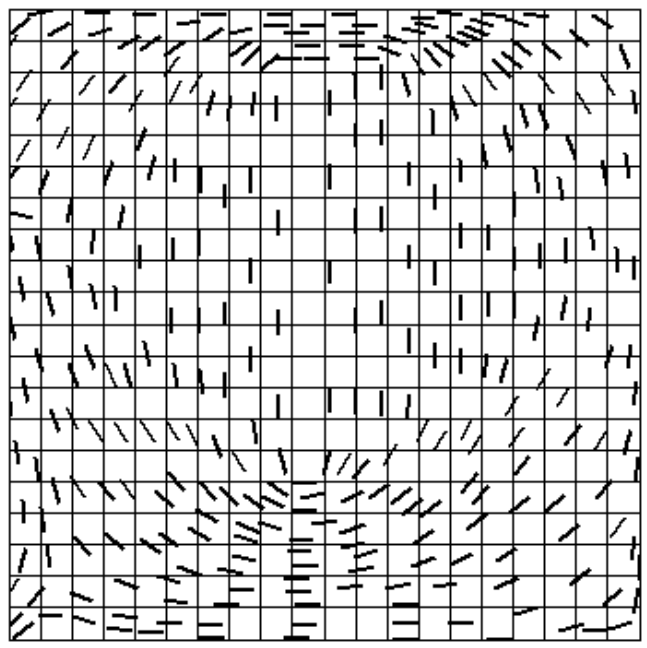

Figure 6: Manually picked data and discretization.

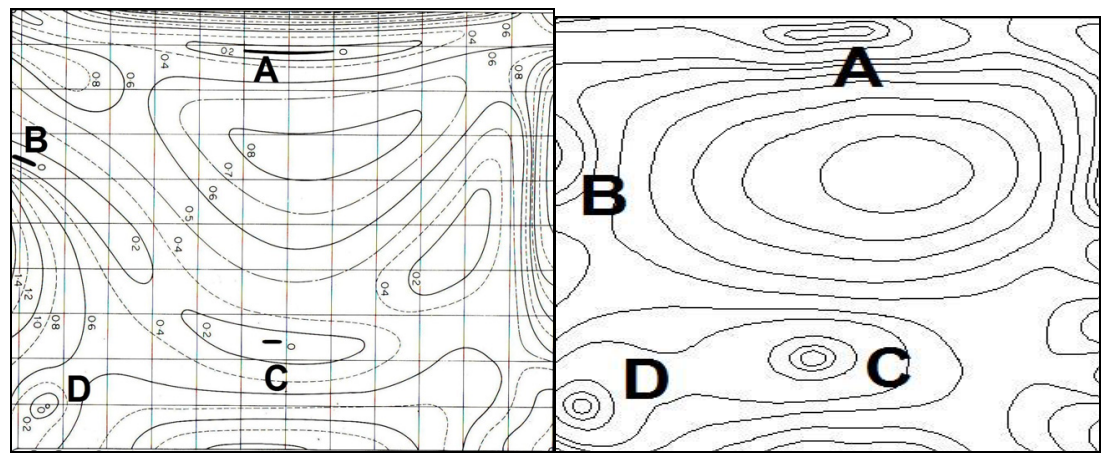

Figure 7: Experimental result for isochromatics [2] (left) and reconstructed isochromatics (right).

The errors are due to approximation used in [2] for plotting of stress trajectories and to manual pick up. The data have been picked from stress trajectories image, using MATLAB software.

Computational domain consists of 400 elements with two collocation points on each interface, Figure 6. The SLAE consisted of 6381 equations and 3200 unknowns. The condition number of this system was $10^{4}$ and the residual was 288.

Isochromatics obtained from photoelasticity and the reconstructed isochromatics are shown in Figure 7 where A,B,C,D are singular (isotropic) points. Experimental and reconstructed stress trajectories are shown in Figure 8. It is remarkable that in this example three singular points $(A, C, D)$ are inside the domain and one B is on its boundary. All of them have been recovered from principal directions data. 


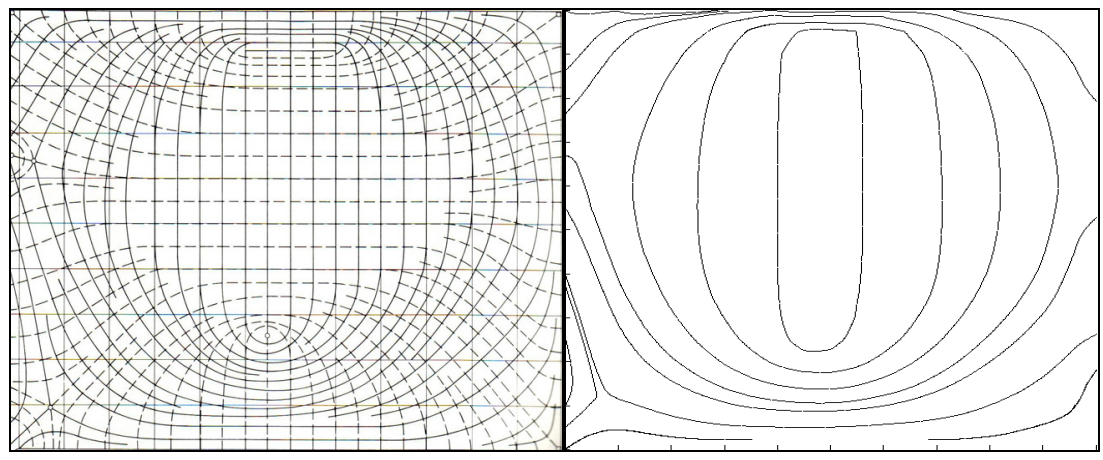

Figure 8: $\quad$ Stress trajectories from experiment [2] (left) and reconstructed stress trajectories (right).

\subsection{Results for Bakelite ring under concentrated diametral loads}

The data from a photoelastic experiment for a ring subjected to the action of two concentrated forces have been used. In this example almost all boundary is free of stresses except of two small loading areas. The stress trajectories image was used to obtain 300 data on principal direction, Figure 9. The stress pattern for compressed circular ring is shown in Figure 10 (left).

Computational domain has been discretized into 320 polar elements, with following distribution: 8 elements placed along radius and 40 elements placed along the circumference with 2 collocation points on each interface. The discretization is shown in Figure 9 (right). The system consisted of 5007 equations and 2560 unknowns. Condition number is 2465 and residual is 176.
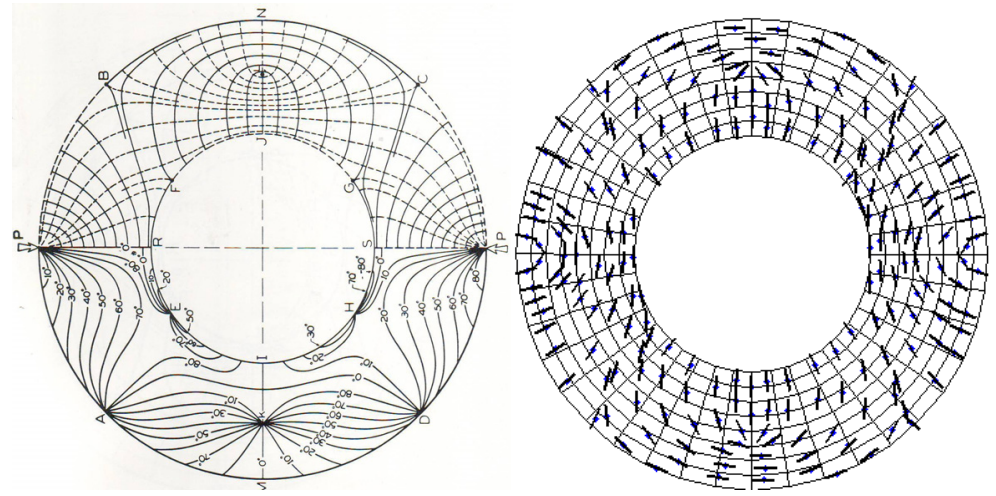

Figure 9: $\quad$ Stress trajectories and isoclinics from [1] (left) and discretization (right).

Reconstructed maximum shear stress is shown in Figure 10 (right). Comparison between photoelasticity isoclinics and reconstructed ones is shown in Figure 11. 

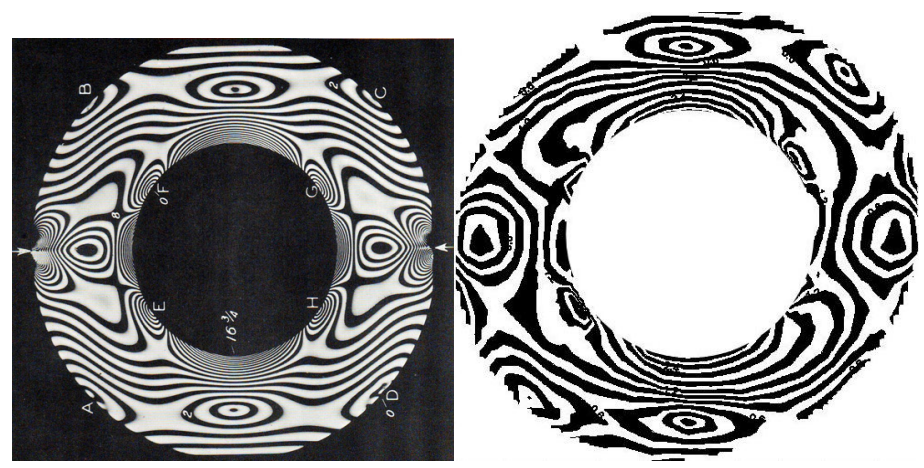

Figure 10: Fringe pattern from [1] (left) and reconstructed fringe pattern (right).
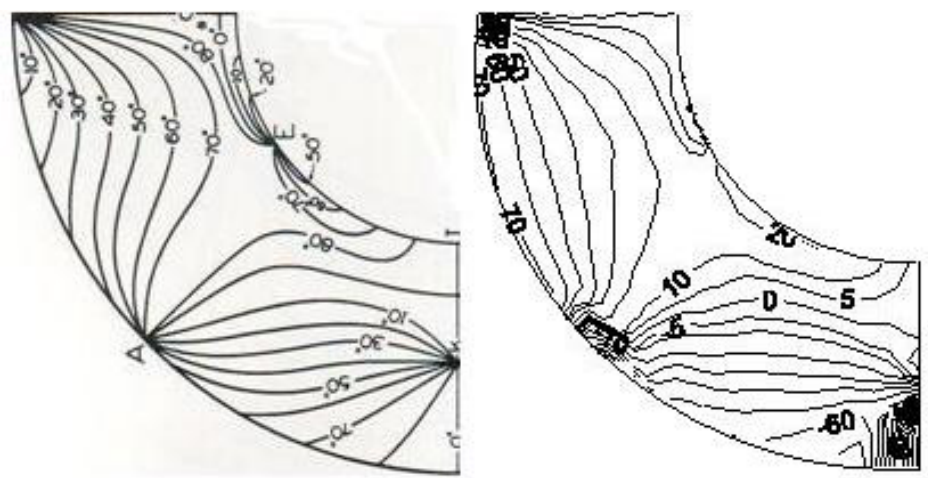

Figure 11: Comparison of isoclinics, photoelasticity (left) and reconstruction (right).

\section{Conclusions}

An approach capable to reconstruct maximum shear stress field from discrete data of principal directions have been proposed. Although the approach is somewhat similar to FEM it does not require consideration of plane elastic boundary value problems and uses approximation of holomorphic functions in subdomains.

The effectiveness of the proposed approach has been confirmed by satisfactory results of reconstruction as synthetic as well as real photoelastic data including identification of internal and boundary singular points.

\section{Acknowledgement}

The authors are grateful to EPSRC for the financial support of this work through the Research Grant EP/E032494/1. 


\section{References}

[1] Frocht M.M., Photoelasticity, vol.1., John Wiley \& Sons Inc.: London, 1962.

[2] Kuske A. and Robertson G., Photoelastic stress analysis, John Wiley \& Sons Ltd.: Bristol, 1974.

[3] Heidbach O., Tingay M., Barth A., Reinecker J., Kurfess D. and Müller B., The 2008 release of the World Stress Map (available online at www.worldstress-map.org).

[4] Galybin A.N. and Mukhamediev Sh.A., Determination of elastic stresses from discrete data on stress orientations, IJSS, 41 (18-19), 5125-5142, 2004.

[5] Mukhamediev Sh.A., Galybin A.N. and Brady B.H.G., Determination of stress fields in the elastic lithosphere by methods based on stress orientations, IJRMMS, 43 (1), 66-88, 2006.

[6] Galybin A.N., Stress fields in joined elastic regions: Modelling based on discrete stress orientations. Int. symposium on In-situ Rock Stress, Trondheim-Norway, 2006.

[7] Muskhelishvili N.I., Some basic problems of the mathematical theory of elasticity, P. Noordhoff Ltd.: Groningen-Holland, 1953.

[8] Irsa J., Galybin A.N., Heat flux reconstruction in grinding process from temperature data, Proc. of the $14^{\text {th }}$ Int. Conf. On Computational Methods and Experimental Measurements, eds. C.A. Brebbia \& G.M. Carlomagno, WIT Press: Southampton, 2009, In print.

[9] Golub G.H. and Van Loan C.F., Matrix computations, The Johns Hopkins Press Ltd.: London, 1996. 